\title{
Hilbert transform is an isometry: A simple proof via real rational functions
}

\author{
Shirley Bromberg Silverstein ${ }^{1}$, Victor A. Cruz Barriguete ${ }^{2}$, and \\ Omar Sanchez Antonio ${ }^{3}$ \\ ${ }^{1}$ Departamento de Matemáticas, Universidad Autónoma \\ Metropolitana, Unidad Iztapalapa, México, E-mail: \\ stbs@xanum.uam.mx \\ ${ }^{2}$ Departamento de Ciencias Básicas, Universidad Autónoma \\ Metropoliana, Unidad Azcapotzalco, México, E-mail: \\ vacb@azc.uam.mx \\ ${ }^{3}$ Instituto de Ciencias Matemáticas, Spain, E-mail: \\ omar.sanchez@icmat.es
}

March 28, 2020

\begin{abstract}
The aim of this note is to give a simple proof of the fact that the Hilbert transform in $\mathcal{L}^{2}(\mathbb{R})$ is an isometry, using a basis for $\mathcal{L}^{2}(\mathbb{R})$ consisting orthonormal real rational functions.
\end{abstract}

\section{Introduction}

The Hilbert transform in $\mathbb{R}$ is the operator

$$
(\mathscr{H} f)(x)=\frac{1}{\pi} P . V . \int_{-\infty}^{\infty} \frac{f(x-y)}{y} \mathrm{~d} y=\frac{1}{\pi} P . V . \int_{-\infty}^{\infty} \frac{f(u)}{x-u} \mathrm{~d} u .
$$

It is well known that $\mathscr{H}: \mathcal{L}^{2}(\mathbb{R}) \rightarrow \mathcal{L}^{2}(\mathbb{R})$ is a bounded linear operator (see e.g. Cotlar [2], Duoandikoetxea [3, 4], Grafakos [5], Laeng [7]). Moreover, it is known that

Theorem 1. The Hilbert transform $\mathscr{H}: \mathcal{L}^{2}(\mathbb{R}) \rightarrow \mathcal{L}^{2}(\mathbb{R})$ is an isometry.

Key words and phrases. Rational functions, Hilbert transform. 2010 Mathematics subject classification. 44A15, 26C15, 44A20 
The usual proof of Theorem 1 uses Fourier transform and Plancherel equality (see e.g. Duoandikoetxea [3], Grafakos [5]).

Recently Duoandikoetxea [4] and Laeng [7] gave alternative proofs without using the Fourier transform. Duoandikoetxea [4] showed that the image of the orthonormal basis of $\mathcal{L}^{2}(\mathbb{R})$ consisting of the Hermite functions by the Hilbert transform is also an orthonormal basis of $\mathcal{L}^{2}(\mathbb{R})$ and therefore the Hilbert transform can be extended to $\mathcal{L}^{2}(\mathbb{R})$ isometrically. Whereas Laeng [7] used approximation of $\mathcal{L}^{2}(\mathbb{R})$ functions by simple functions and then used the norm of the Hilbert transform of the characteristic function of an interval.

In this work, we use the basis for $\mathcal{L}^{2}(\mathbb{R}) \mathscr{B}$ defined in [1] and, by calculating the Hilbert transform of the elements of $\mathscr{B}$, we obtain a simple proof of Theorem 1.

This note is organized as follows. In Section 2, we present the preliminaries needed to develop the result. In Section 3 we prove Theorem 1 . The last section, Section 4 , is devoted to address technical details of the proof and other auxiliary lemmas.

\section{Preliminaries}

We will denoted by $\mathbb{N}=\{0,1,2, \ldots\}$ and denote by $\mathbb{R}$ and $\mathbb{C}$ the set of the real and complex numbers respectively. Christov showed in [1] (see also [6, pp. 63]) that the family $\mathscr{B}=\left\{S_{n}, C_{n}\right\}_{n \in \mathbb{N}}$ where,

$$
S_{n}(x)=\sqrt{\frac{2}{\pi}} \frac{(-1)^{n+1}}{\left(x^{2}+1\right)^{n+1}} \sum_{k=0}^{n}(-1)^{k}\left(\begin{array}{l}
2 n+1 \\
2 k+1
\end{array}\right) x^{2 k+1}
$$

and

$$
C_{n}(x)=\sqrt{\frac{2}{\pi}} \frac{(-1)^{n+1}}{\left(x^{2}+1\right)^{n+1}} \sum_{k=0}^{n}(-1)^{k+1}\left(\begin{array}{c}
2 n+1 \\
2 k
\end{array}\right) x^{2 k}
$$

is a complete orthonormal basis for $\mathcal{L}^{2}(\mathbb{R})$. The Fourier series of $f \in \mathcal{L}^{2}(\mathbb{R})$, with respect to $\mathscr{B}$, is given by

$$
f=\sum_{n \in \mathbb{N}} a_{n} S_{n}+b_{n} C_{n}
$$

where the corresponding Fourier coefficients are determined by

$$
a_{n}=\left\langle f, S_{n}\right\rangle, \quad b_{n}=\left\langle f, C_{n}\right\rangle .
$$

With this representation of $f$, the $\mathcal{L}^{2}(\mathbb{R})$ norm of $f$ is given by the Fourier coefficients and the Plancherel equality:

$$
\|f\|_{2}^{2}=\sum_{n \in \mathbb{N}} a_{n}^{2}+b_{n}^{2} .
$$


To end the section, we remind the reader that the Hilbert transform $\mathscr{H}$ is a bounded linear operator of $\mathcal{L}^{2}(\mathbb{R})$. Now we can proceed to the proof of the main Theorem.

\section{Proof of Theorem 1}

The proof of Theorem 1 is really simple using the complete orthonormal basis $\mathscr{B}$ of $\mathcal{L}^{2}(\mathbb{R})$ and the following lemma that states that the Hilbert transform acts as a kind of involution on $\mathscr{B}$. More precisely

Lemma 1. For every $n \in \mathbb{N}$,

$$
\mathscr{H} S_{n}=C_{n} \quad \text { and } \quad \mathscr{H} C_{n}=-S_{n} .
$$

We now turn to the proof of the principal result.

Proof of Theorem 1. Given $f \in \mathcal{L}^{2}(\mathbb{R})$, and since $\mathscr{B}$ is a complete orthonormal basis and the Hilbert transform is a bounded linear operator, we have

$$
\mathscr{H} f=\sum_{n \in \mathbb{N}} a_{n} \mathscr{H} S_{n}+b_{n} \mathscr{H} C_{n},
$$

where $a_{n}$ y $b_{n}$ are, as before, the Fourier coefficients of $f$ with respect to $\mathscr{B}$. By Lemma $1 \mathscr{H} S_{n}=C_{n}$ and $\mathscr{H} C_{n}=-S_{n}$, hence

$$
\mathscr{H} f=\sum_{n \in \mathbb{N}} a_{n} C_{n}-b_{n} S_{n} .
$$

Finally by the Plancherel equality we conclude

$$
\|\mathscr{H} f\|_{2}^{2}=\sum_{n \in \mathbb{N}} a_{n}^{2}+b_{n}^{2}=\|f\|_{2}^{2} .
$$

\section{Auxiliary results}

To prove Lemma 1 we introduce the family $\mathscr{D}=\left\{\phi_{n}\right\}_{n \in \mathbb{N}}$ where

$$
\phi_{n}:=C_{n}+i S_{n}, n \in \mathbb{N} .
$$

Notice that $\phi_{n}$ takes complex values so that we are working in $\mathcal{L}^{2}(\mathbb{R}, \mathbb{C})$ which is a Hilbert space with inner product

$$
\langle f, g\rangle=\int_{-\infty}^{\infty} f(x) \overline{g(x)} \mathrm{d} x
$$

for $f, g \in \mathcal{L}^{2}(\mathbb{R}, \mathbb{C})$. Also for $f \in \mathcal{L}^{2}(\mathbb{R}, \mathbb{C})$,

$$
\mathscr{H} f=\mathscr{H} \operatorname{Re}(f)+i \mathscr{H} \operatorname{Im}(f) .
$$




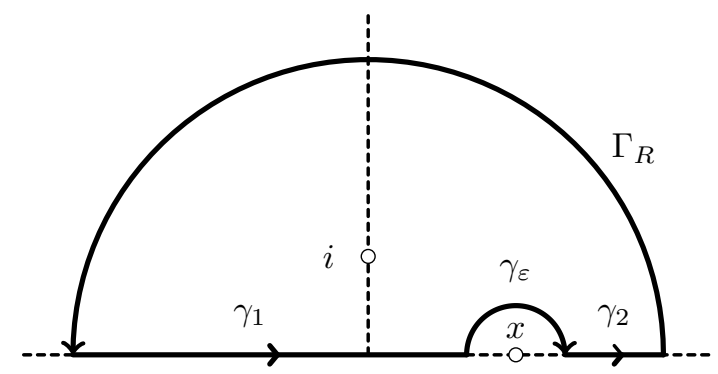

Figure 1: The contour $\Gamma_{R}$

Proof of Lemma 1 A direct calculation shows that

$$
\phi_{n}(x)=-i \sqrt{\frac{2}{\pi}} \frac{(x+i)^{n}}{(x-i)^{n+1}} .
$$

Then

$$
\left(\mathscr{H} \phi_{n}\right)(x)=\frac{-i}{\pi} \sqrt{\frac{2}{\pi}} \text { P.V. } \int_{-\infty}^{\infty} \frac{(u+i)^{n}}{(u-i)^{n+1}(x-u)} \mathrm{d} u .
$$

Let

$$
\Phi_{n}(w):=\frac{\phi_{n}(w)}{x-w}=-i \sqrt{\frac{2}{\pi}} \frac{(w+i)^{n}}{(w-i)^{n+1}(x-w)}
$$

for $x \in \mathbb{R}$. The function $\Phi_{n}(w)$ is a meromorphic function in the upper half plane with a pole of order $n+1$ at $w=i$ and a simple pole at $w=x$. Consider the contour $\Gamma_{R}$ shown in Figure 1, that contains the pole $i$ in its interior and avoids the pole at $w=x$. Then on the one hand we have

$$
\int_{\Gamma_{R}} \Phi_{n}(w) \mathrm{d} w=2 \pi i \operatorname{Res}\left(\Phi_{n}(w), w=i\right)
$$

and on the other

$$
\int_{\Gamma_{R}} \Phi_{n}(w) \mathrm{d} w=\left(\int_{\gamma_{R}}+\int_{\gamma_{1}}+\int_{\gamma_{\varepsilon}}+\int_{\gamma_{2}}\right) \Phi_{n}(w) \mathrm{d} w .
$$

Since on $\gamma_{R}$

$$
\left|\Phi_{n}(w)\right|<\sqrt{\frac{2}{\pi}}\left(\frac{R+1}{R-1}\right)^{n} \frac{1}{(R-1)|R-| x||} \approx \sqrt{\frac{2}{\pi}} \frac{1}{R^{2}},
$$

then

$$
\lim _{R \rightarrow \infty} \int_{\gamma_{R}} \Phi_{n}(w) \mathrm{d} w=0
$$


We also have that

$$
\lim _{\substack{\varepsilon \rightarrow 0 \\ R \rightarrow \infty}}\left(\int_{\gamma_{1}} \Phi_{n}(w) \mathrm{d} w+\int_{\gamma_{2}} \Phi_{n}(w) \mathrm{d} w\right)=P . V . \int_{-\infty}^{\infty} \Phi_{n}(w) \mathrm{d} w .
$$

To evaluate the remaining integral on the right hand side of equation (2), note that the function we are integrating is uniformly continuous near $x-i$ then

$$
\lim _{\varepsilon \rightarrow 0} \int_{\gamma_{\varepsilon}} \Phi_{n}(w) \mathrm{d} w=\sqrt{\frac{2}{\pi}} \int_{0}^{\pi} \lim _{\varepsilon \rightarrow 0} \frac{\left(x+i-\varepsilon e^{-i t}\right)^{n}}{\left(x-i-\varepsilon e^{-i t}\right)^{n+1}} \mathrm{~d} t=i \pi \phi_{n}(x) .
$$

Hence

$$
\int_{\Gamma_{R}} \Phi_{n}(w) \mathrm{d} w \rightarrow P . V . \int_{-\infty}^{\infty} \frac{\phi_{n}(w)}{x-w} \mathrm{~d} w+i \pi \phi_{n}(x),
$$

as $R \rightarrow \infty$ and $\varepsilon \rightarrow 0$. The proof will be complete as soon as we compute the residue of $\Phi_{n}$ around $w=i$. The expansion of $\Phi_{n}(w)(w-i)^{n+1}$ around $w=i$ gives

$$
\Phi_{n}(w)(w-i)^{n+1}=-i \sqrt{\frac{2}{\pi}} \sum_{k=0}^{n}\left(\begin{array}{l}
n \\
k
\end{array}\right) \frac{(2 i)^{n-k}}{x-i} \sum_{j=0}^{\infty} \frac{(w-i)^{j+k}}{(x-i)^{j}} .
$$

Hence

$$
\operatorname{Res}\left(\Phi_{n}(w), w=i\right)=-i \sqrt{\frac{2}{\pi}} \sum_{k=0}^{n}\left(\begin{array}{l}
n \\
k
\end{array}\right) \frac{(2 i)^{n-k}}{(x-i)^{n-k+1}}=\phi_{n}(x) .
$$

From (3), (4) and (5) we get

$$
\mathscr{H} \phi_{n}=2 i \phi_{n}-i \phi_{n}=i \phi_{n} .
$$

Finally

$$
\mathscr{H} C_{n}=\operatorname{Re}\left(\mathscr{H} \phi_{n}\right)=-S_{n}, \quad \mathscr{H} S_{n}=\operatorname{Im}\left(\mathscr{H} \phi_{n}\right)=C_{n} .
$$

\section{$5 \quad$ Final remarks}

The proof we present not only is quite simple but has the advantage that it requires elementary results. On the other hand, the elements in the family are $\mathrm{C}^{\infty}$ rational functions that behave like $1 /|x|$ at $\pm \infty$.

\section{References}

[1] C. I. Christov, A complete orthonormal system of functions in $L^{2}(-\infty, \infty)$ space, SIAM J. Appl. Math., vol. 42, no. 6, 1982, pp. 1337-1344, DOI $10.1137 / 0142093$. 
[2] M. Cotlar, A combinatorial inequality and its applications to $L^{2}$-spaces, Rev. Mat. Cuyana, vol. 1, 1955, pp. 41-56.

[3] J. Duoandikoetxea, Fourier analysis, American Mathematical Society, GSM, vol. 29, pp. xviii+222, 2001.

[4] J. Duoandikoetxea, The Hilbert transform and Hermite functions: a real variable proof of the $L^{2}$-isometry, J. Math. Anal. Appl., vol. 347, no. 2, 2008, pp. 592-596, DOI 10.1016/j.jmaa.2008.06.016.

[5] L. Grafakos, Classical Fourier analysis, GTM, vol. 249, Springer, third ed., 2014.

[6] J. R. Higgins, Completeness and basis properties of sets of special functions, Cambridge University Press, 1977.

[7] E. Laeng, A simple real-variable proof that the Hilbert transform is an $L^{2}$ isometry, C. R. Math. Acad. Sci. Paris, vol. 348, no. 17-18, 2010, pp. 977980, DOI 10.1016/j.crma.2010.07.002. 